\title{
Retroactive interference in rat radial maze performance: The role of point of delay interpolation and the similarity and amount of interpolated material
}

\author{
ROBERT G. COOK and MICHAEL F. BROWN \\ University of California, Berkeley, California
}

\begin{abstract}
The conditions necessary for producing retroactive interference (RI) were examined in a 12 arm radial maze. Rats were first given either three or nine forced choices in a to-be-remembered maze. During a 2-h delay, they received one or two trials in a second 12-arm maze, located either in a different room or the same room as the to-be-remembered maze. During the postdelay memory test, RI from the interference trials was produced only when nine choices had been made in the to-be-remembered maze and two interference trials had been conducted during the delay interval. RI was not found when only three forced choices had to be retained or after a single interference trial. The similarity between the interpolated and to-be-remembered mazes had no effect on choice accuracy. It was concluded that two conditions are required for the production of RI in the radial maze. First, a "large amount" of information should be resident in working memory. Second, a substantial number of interpolated trials or choices must be made during the delay.
\end{abstract}

Recent studies have suggested that the memory processes mediating the performance of rats in the radialarm maze differ from those observed in other species and tasks. When compared with the performance of pigeons in the matching-to-sample task, for example, rats seem to be able to retain more information (Olton, Collison, \& Werz, 1977; Roberts, 1979) for a longer period of time (Beatty \& Shavalia, 1980a, 1980b) and with a great degree of accuracy and a minimum of proactive or retroactive interference (Beatty \& Shavalia, 1980a, 1980b; Maki, Brokofsky, \& Berg, 1979; Olton, 1978; Roberts, 1981). Their performance is often considered remarkable (Beatty \& Shavalia, 1980b; Magni, Krekule, \& Bureš, 1979; Roberts, 1981), and has led to claims that "spatial memory" is well developed in the rat, may suffer no decay with time (Olton, 1978), and may have an unlimited capacity (Roberts, 1984).

The notion that the processing of memories in the radial maze is different from other tasks has resulted, in part, from experiments investigating retroactive interference (RI). Beatty and Shavalia (1980a, 1980b) and Maki et al. (1979) failed to find any evidence for RI with memories for choices in the radial maze, even though many of the

The conduct and preparation of this research was supported by NSF Grant BNS-8317577 to D. A. Riley and by NRSA postdoctoral grants (5 T32 EY-07024 and 1 FY EY-05653-01A1) from the National Eye Institute to the first author. The authors thank D. A. Riley for his comments and encouragement during the conduct of this research, A. A. Wright and two anonymous reviewers for their helpful comments on an earlier version of this manuscript, and Jackie Rivera for her artwork on the figures. Reprint requests should be sent to: Robert G. Cook, University of Texas Health Science Center, Sensory Sciences Center, 6420 Lamar Fleming Ave., Texas Medical Center, Houston, TX 77030. interfering materials were known to produce $\mathrm{RI}$ in other species and tasks (Cook, 1980; D’Amato \& O'Neill, 1971; Grant \& Roberts, 1976; Herman, 1975). Maki et al. (1979) tested retention of four choices in an 8-arm radial maze following interpolation of illumination change, food, auditory material, and a trial in another radial maze. None of these interpolated materials decreased subsequent choice accuracy. Beatty and Shavalia (1980a, 1980b) were also unable to produce RI under more severe conditions. Beatty and Shavalia (1980a) tested rats with two 8-arm mazes. After four choices on maze A, four choices were conducted on maze B. Subsequently, the rats were returned to each maze in turn. Postdelay accuracy in maze A was not affected by the interpolation of maze B, despite the fact that information about maze $B$ choices had to be retained for its subsequent test. Beatty and Shavalia (1980b) found that narcotizing doses of barbiturate anesthetics during the delay also failed to produce RI.

Demonstrations of RI come from two studies. Shavalia, Dodge, and Beatty (1981) found that electroconvulsive shock (ECS), when delivered at $30 \mathrm{~min}$, but not at 0 or $15 \mathrm{~min}$, would reduce subsequent performance into a 4-6-h retention interval. This is an interesting exception to the usual monotonically decreasing temporal gradient of ECS effects on memory (Duncan, 1949). Roberts (1981) demonstrated that three interpolated trials, each conducted in separate radial mazes, would produce RI when interpolated after the fourth choice of an 8-arm maze.

The experiment reported in this article examines the necessary conditions for producing RI in the radial maze. Previous failures may have suffered from a "ceiling effect" as a result of the use of task parameters that failed 
to tax the rat's processing capacity. To increase task difficulty, a 12-arm maze was used in the present experiment. In addition, several factors were manipulated that were expected to affect the degree of RI. These consisted of varying (1) the point in the choice sequence at which the material was interpolated, (2) the amount of interpolated material, and (3) the similarity of the interpolated material to the to-be-remembered material. The variation of the point in the choice sequence at which a 2 -h retention interval was interpolated was designed to manipulate memory load. Rats were stopped after either the third or ninth choice in the to-be-remembered maze. Regardless of the size of the memory unit, later interpolation of the delay into the choice sequence should require more choices to be retained than a delay interpolated after only a few choices, assuming that choices are retained in the form of a retrospective code (Cook, Brown, \& Riley, 1985).

The amount and similarity of the interpolated material was varied by conducting zero, one, or two interpolated trials in another radial maze. This second maze was located either in a completely different room (designated room 2) or in a slightly displaced and overlapping location of the room in which the to-be-remembered maze was normally located (room 1). When interpolated trials were conducted in room 1 , the to-be-remembered maze was removed.

\section{METHOD}

\section{Subjects}

The subjects were eight Sprague-Dawley rats maintained at $80 \%$ of their free-feeding weights. They were housed in individual cages with free access to water. The sessions were conducted during the first $4 \mathrm{~h}$ of their dark phase (12:12 LD cycle). The subjects had had extensive experience with the radial-arm maze and required little preliminary training.

\section{Apparatus}

Two radial arm mazes were used. Their design consisted of 12 arms radiating out of a $41-\mathrm{cm}$ center platform, $61 \mathrm{~cm}$ above the floor. Each arm was $80 \mathrm{~cm}$ long and $10 \mathrm{~cm}$ wide and had a stainless steel food cup located at the end. The entrance to each arm was through a 7.5 -cm-diam hole in a metal barrier that surrounded the center platform. The arms of each maze were hinged to the center platform, allowing the mazes to be moved.

There were several differences between the two mazes. In maze $\mathrm{A}$, located in room 1 and used as the to-be-remembered maze, the entrance to the arms could be controlled by either individual guillotine doors or by a large circular guillotine door that allowed access to all arms simultaneously. The clockwise side of each arm in maze A had a $12.5-\mathrm{cm}$-high, $26-\mathrm{cm}$-long barrier to prevent the animals from crossing over to arms without first returning to the center platform. The second maze was designated maze $\mathrm{A}^{\prime}$ when in room 1 , because its location was displaced $2 \mathrm{~m}$ from where the center platform of maze A was normally located, and maze B when it was located in room 2. This maze was used as the interference maze. Maze $A^{\prime} / B$ differed from maze $A$ in the following ways: (1) Only a circular guillotine door was used to control access to the arms; (2) each clockwise barrier was $62 \mathrm{~cm}$ long $\times 12.5 \mathrm{~cm}$ high, except for the last $38 \mathrm{~cm}$. which extended down the length of each arm and was $7.5 \mathrm{~cm}$ high.
The two rooms used in the experiment were distinct, at least to the experimenters. The primary features of room 1 were a large furnace, a rack of cages, a large white screen, and a large black plastic sheet along one side of the room. All these features were from 2 to $3 \mathrm{~m}$ from the maze. Room 2 was smaller and had highcontrast posters on two of the four walls. A large ventilation duct was located near a third wall. Three of the walls of room 2 were about $1 \mathrm{~m}$ from the maze.

\section{Procedure}

Initial training. The goal of initial training was to familiarize the subjects with changes in the procedure from their previous experience. These changes consisted of the use of the second maze (maze $A^{\prime} / B$ ) and the use of a forced-choice procedure in maze $A$. All previous training had been conducted in maze $A$.

During training, the subjects were given two trials per day. The first trial of each day was conducted in maze A. Two 45-mg food pellets (Noyes) were placed in the food cup at the end of each arm. The subject was placed on the center platform, and the door to a single arm was then opened. When the subject returned to center platform, the door was lowered and the door to a second arm was opened. This procedure was repeated until six arms had been visited. All forced choices were randomly predetermined by the experimenters. The subject was then removed and placed in an individual holding cage. During a 30 -min delay, the individual doors were replaced by the large circular door. The subject was then returned to maze $\mathrm{A}$, the door was opened to provide access to all $12 \mathrm{arms}$, and the subject was allowed to make choices until the remaining food was found or until 20 total choices had been made, with the latter number including the predelay forced choices.

The second trial of each day occurred $1 \mathrm{~h}$ later. It was conducted with maze $A^{\prime} / B$. Training trials were conducted in room 1 (maze $A^{\prime}$ ) or room 2 (maze $B$ ) in an alternating fashion over days. The subject was placed on the center platform and was allowed 20 choices or until all 12 arms had been visited.

Training continued on maze A, maze $A^{\prime}$, and maze $B$ until a subject had made 10 correct choices in its first 12 choices and completed the entire maze in 15 or fewer choices on three out of four consecutive trials for each maze and location. One subject never met this criterion and was dropped from the experiment. The mean number of sessions to this criterion for the remaining seven subjects was: maze $\mathrm{A}=4.6$; maze $\mathrm{A}^{\prime}=5.1$; maze $\mathrm{B}=6.3$.

RI testing. RI testing began on the day after a subject reached criterion on all three mazes. The subject was placed in the center platform of maze A and directed to either three or nine arms, using the forced-choice procedure. Following the last forced choice, the subject was removed and placed in its holding cage.

During a 2-h delay, the subject received one of four RI or four control treatments. During RI treatments, the subjects received one or two interpolated trials in room 1 (maze $A^{\prime}$ ) or room 2 (maze $B$ ). RI treatments with one interpolated trial were conducted 50 min after the subject was removed from maze A; RI treatments with two interpolated trials had the first trial at $50 \mathrm{~min}$ and the second trial at $65 \mathrm{~min}$ after removal. The control treatments were as similar as possible to the RI treatments, except for the placement in the interference maze. During the delay of control treatments, the subject was moved to the room scheduled for the interpolated trials. While there, the subject was given 24 or 48 pellets at the same time(s) as the subjects being treated with interpolated trials.

Following the 2-h delay, the subject was returned to maze $A$ and given a memory test. The circular guillotine door was raised and the subject was allowed free access to its unfinished maze. Choices were allowed until all correct arms had been chosen or until 20 total choices had been made.

Four blocks of RI testing were conducted. A block consisted of 12 different trials. Eight trials of each block were RI treatments, composed of the orthogonal combination of three factors: point of 
delay interpolation (three or nine forced choices), number of interpolated trials (one or two), and the maze in which these interpolated trials were conducted (maze $\mathbf{A}^{\prime}$ or maze $\mathrm{B}$ ). Four trials of each block were control treatments composed of the orthogonal combination of two factors: point of delay interpolation and whether the subject was moved to room 1 or room 2 . The amount of food ( 24 or 48 pellets) received during the delay was counterbalanced across blocks. The room in which the interpolated trials were conducted was counterbalanced using a $\mathrm{A}^{\prime} \mathrm{BBA}^{\prime}$ scheme. Within this constraint, the order of treatments was random for each subject.

\section{RESULTS}

The mean number of total choices required per session to finish maze A for the different treatment conditions is displayed in Figure 1. Analysis of the delay-interval feeding conditions revealed no significant differences and are combined in Figure 1 and all subsequent analyses. Examination of Figure 1 suggests that the different rooms in which the interpolated trials were conducted had little or no effect on maze A choice accuracy. However, the number of interpolated trials during the delay interval did appear to have an effect on choice accuracy in the late interpolation conditions, but not in the early interpolation conditions.

Each interpolation condition was analyzed separately, because the opportunities to make errors following the delay are not the same in the early and late interpolation conditions (see Cook et al.). Different repeated measures analyses of variance (ANOVAs; number of interpolated trials $\times$ location of interpolated trials $\times$ subjects) were conducted for the early and late interpolated conditions. These revealed that for neither interpolation condition was there an effect of location $[F s(1,6)<1.5]$. The number of interpolated trials did have a significant effect on choice

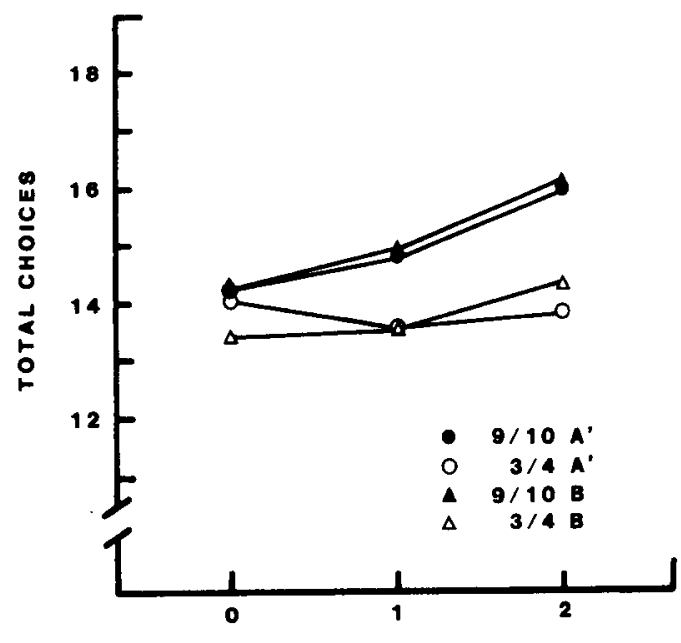

MUMBER OF INTERPOLATED TRIALS

Figure 1. The mean number of choices per session taken to finish maze $A$ during $R I$ testing as a function of point-of-delay interpolation $(3 / 4=$ delay after choice $3 ; 9 / 10=$ delay after choice 9$)$, similarity of interpolated material ( $A^{\prime}$ or $B$ ), and number of trials in the interpolated maze.

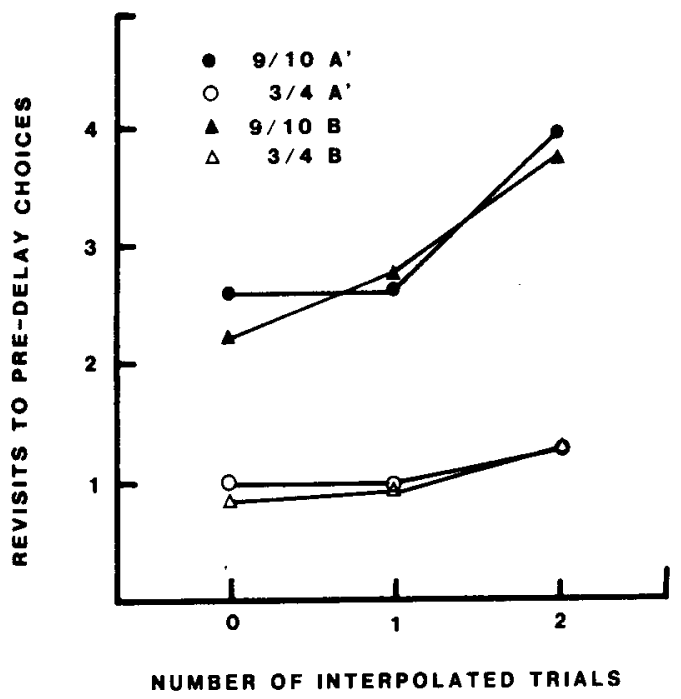

Figure 2. The mean number of predelay choices revisited per session during RI testing as a function of point of interpolation (3/4 $=$ delay after choice $3 ; 9 / 10=$ delay after choice 9 ), similarity of interpolated material ( $A^{\prime}$ or $B$ ), and number of trials in the interpolated maze.

accuracy in the late interpolation conditions $[\mathrm{F}(2,12)=$ $6.6, p<.01]$, but there was no corresponding effect for number of interpolated trials in the early interpolation condition $[\mathrm{F}(2,12)=1.1]$.

The mean number of predelay arms revisited during postdelay testing in maze A is displayed in Figure 2 for the different treatment conditions. Analysis of these predelay intrusions was conducted because they measure most clearly the retroactive effect of the interpolated trials. These data show the same pattern of results as Figure 1. This was confirmed by two ANOVAs analogous to those described above. No effect (Fs $<1.5$ ) of interpolated maze location was found in either the early or late interpolation conditions. A significant effect of the number of trials in the interpolated maze was obtained in the late interpolation conditions $[\mathrm{F}(2,12)=5.6, \mathrm{p}<.02]$, but not in the early interpolation condition $[\mathrm{F}(2,12)=2.3]$.

Displayed in Figure 3 are the serial positions in the initial choice sequence of these predelay intrusions for the late interpolation conditions. The mean number of revisits per session to predelay arms observed during postdelay testing are collapsed across three successive choices and the location of the interpolated trials. The increase in RI produced by the interpolation of two trials appears to be equivalent for all serial positions when compared with the control condition. A small RI effect after one interpolated trial seems to be restricted only to arms chosen earlier in the choice sequence. An ANOVA (number of interpolated trials $\times$ serial position $\times$ subject) revealed a significant main effect for choice serial position $[F(2,12)=$ $8.6, p<.005]$ and number of interpolated trials $[\mathrm{F}(2,12)$ $=7.2, \mathrm{p}<.009]$, but no interaction between these factors. 


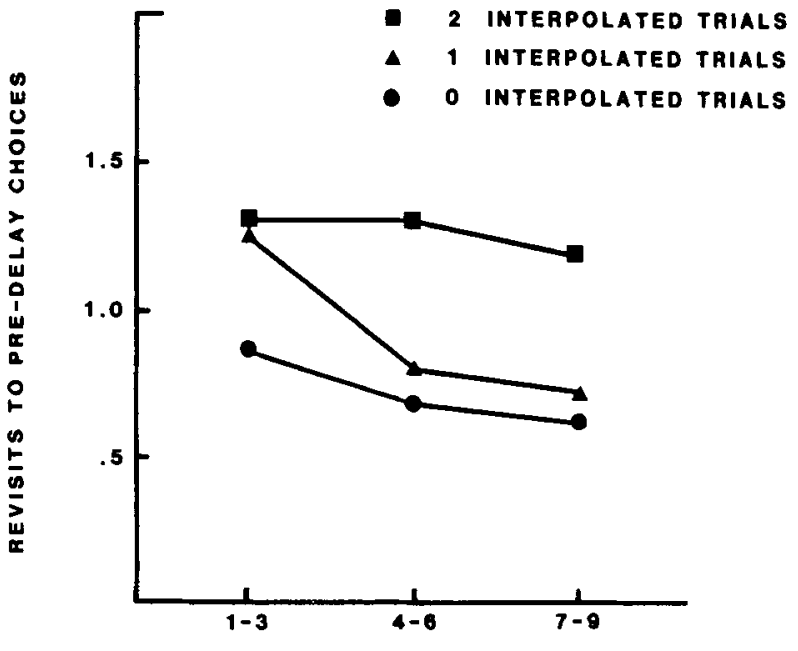

CHOICE SERIAL POSITION

Figure 3. Mean number of predelay choices revisited per session during RI testing following a delay in the $9 / 10$ conditions as a function of their initial choice serial position. All treatments are collapsed across similarity conditions.

\section{DISCUSSION}

This experiment clarifies the conditions required for the production of retroactive interference in the retention of previous choices by rats in a radial maze. Two conditions were necessary, but neither was sufficient, to produce RI in the experiment. The first requirement is that many prior choices be resident in working memory during the presentation of the interpolated material. The second requirement is the interpolation of a substantial amount of interpolated material.

The difference in the pattern of results for material interpolated early and late in the choice sequence of the tobe-remembered maze indicates that memory load is an important factor in the production of RI. Interference is seen when trials are interpolated after the ninth choice, where memory load is presumably "large," but not after the third choice, where memory load should be "smaller." Previous failures to observe RI may have arisen from the use of an eight-arm maze with a delay interpolated after the fourth choice, a condition that is most similar to those in which no RI was detected in the experiment reported here.

The results discredit an alternative explanation for the effect of point of interpolation, in which the rats are not remembering the nine predelay arms in the late conditions, but instead are recoding their predelay experience into the three arms remaining to be visited. This would be a type of prospective code (Honig \& Thompson, 1982; Riley, Cook, \& Lamb, 1981; Roitblat, 1980). The implication of this explanation would be that the interpolated material is effective only when a prospective code is being used. While such an alternative is intriguing, it appears not to be the case in the present experiment. If rats were prospectively coding in the late interpolation conditions, one might expect the performance to be equivalent with the early interpolation conditions, since presumably the memory load would be the equal (Cook et al., 1985). In the current experiment, however, the late interpolation control conditions produced poorer performance than the early interpolation control conditions. A second reason for discounting a prospective account of the experiment stems from other experiments by the authors in which retention interval and point of interpolation were systematically varied (Cook et al., 1985). Those experiments indicated that after a delay of $1 \mathrm{~h}$ the rats used only retrospective information, or at least failed to use prospective information, in making postdelay choices. For the experiment reported in this article, the 2 -h retention interval was specifically used to prevent the possibility of prospective coding. Finally, the presence of serial position effects in the late interpolation conditions provides additional evidence that only retrospective memory was being used (Cook et al., 1985).

The second factor important for the production of RI was the amount of interpolated material. Two studies have now produced RI using trials in another radial maze as the source of the interpolated material. Roberts (1981) reported that trials in three different interpolated mazes were needed for the detection of RI. The results of our experiment confirm and extend his findings to a maze with more arms, to the repetition of the interpolated trials within the same interpolated maze, and to a superior forced-choice procedure. The results also make clear, however, that the mere insertion of a large number of interpolated trials is not sufficient to produce RI, as demonstrated by the lack of RI in the early interpolation conditions with two interpolated trials.

Examination of the serial positions for the RI and control conditions indicated that the increase in errors in the two-trial conditions was due to the fact that more errors were made at all choice serial positions. In the one-trial conditions, the increase in errors seemed confined to the first three serial positions. This suggests that the effect of the interfering trials was to displace items in memory, with earlier choices being eliminated first, as indicated by the slight increase in errors in the serial position curve for the one-trial condition. When the number of interpolated choices is increased, later choices from the to-beremembered maze are displaced. There was no statistical support, however, for an interaction between serial position and number of interpolated trials, and as a result judgment will have to be suspended on this possibility.

It has been expected that the similarity between to-beremembered and interpolated trials conducted in the same room (room 1) would maximize confusion and reduce performance. The results clearly indicate that this did not occur. Roberts (1981) also failed to detect any effect of interpolated maze similarity on retention. Roberts placed an interference maze directly adjacent to or on top of the to-be-remembered maze. After four choices on the to-beremembered maze, four forced choices were made on the 
other maze during the delay. There was no evidence of $\mathrm{RI}$ when the rats were subsequently required to finish the to-be-remembered maze. Roberts could produce interference only when the rats were placed on the arms of the to-be-remembered maze during the delay and then subsequently required to finish the maze, disregarding these interpolated placements.

Our experiment also failed to detect an influence of similarity in any condition, including those that produced RI. Studies of longer term retention have found that different contexts can provide important "protection" for conflicting learning experiences and allow animals to discriminate similar stimuli (Spear et al., 1980; Zentall, 1970). Could the slight displacement of the interpolated maze in room 1 have been sufficient to prevent similarity from having an effect on choice accuracy? If the rat's acuity for detecting slight differences in spatial location is very good, this spatial acuity may help to segregate similar information in memory into different "contexts" and protect it from subsequently encoded information.

The experiment reported here suggests that the conflicting evidence concerning RI in the radial maze was due, in part, to the simplicity of the task and type of the interpolated material previously tested. When the difficulty of the task is increased and a greater demand is placed on the rat's memory capacity, RI can be produced when a substantial number of choices are interpolated into the retention interval. Thus, the evocation of a different memory process for rats when tested in the radial-arm maze, at least as indicated by the previous difficulty in detecting RI, appears to be unnecessary.

\section{REFERENCES}

Beatty, W. W., \& Shavalia, D. A. (1980a). Rat spatial memory: Resistance to retroactive interference at long retention intervals. Animal Learning \& Behavior, 8, 550-552.

Beatty, W. W., \& Shavalia, D. A. (1980b). Spatial memory in rats: Time course of working memory and effect of anesthetics. Behavioral and Neural Biology, 28, 454-462.

Cook, R. G. (1980). Retroactive interference in pigeon short-term memory by a reduction in ambient illumination. Journal of Experimental Psychology: Animal Behavior Processes, 6, 326-338.

Cook, R. G., Brown, M. F., \& Riley, D. A. (1985). Flexible memory processing by rats: Use of prospective and retrospective information in the radial maze. Journal of Experimental Psychology: Animal Be. havior Processes, 11, 453-469.

D'AmAto, M. R., \& O'NeILL, W. (1971). Effect of delay-interval illumination on matching behavior in the capuchin monkey. Journal $O$ Experimental Psychology, 15, 327-333.

Duncan, C. P. (1949). The retroactive effect of electroshock on learning. Journal of Comparative and Physiological Psychology, 42, 32-44.

Grant, D. S., \& RoberTs, W. A. (1976). Sources of retroactive inhibition in pigeon short-term memory. Journal of Experimental Psychology: Animal Behavior Processes, 2, 1-16.

Herman, L. M. (1975). Interference and auditory short-term memory in the bottle-nosed dolphin. Animal Learning \& Behavior, 3, 43-48

Honig, W. K., \& Thompson, R. K. R. (1982). Retrospective and prospective processing in animal working memory. The Psychology of Learning and Motivation, 16, 239-283.

MAGNI, S., Krekule, I., \& Bureš, J. (1979). Radial maze type as a determinant of the choice behavior of rats. Journal of Neuroscience Methods, 1, 343-352.

MAKI, W. S., BrokofSKY, S., \& BERG, B. (1979). Spatial memory in rats: Resistance to retroactive interference. Animal Learning \& Be. havior, 7, 25-30.

OLton, D. S. (1978). Characteristics of spatial memory. In S. H. Hulse, H. Fowler, \& W. K. Honig (Eds.), Cognitive processes in animai behavior. Hillsdale, NJ: Erlbaum.

Olton, D. S., Collison, C., \& Werz, M. A. (1977). Spatial memory and the radial arm maze performance of rats. Learming and Motiva. tion, 8, 289-314.

Riley, D. A., COOK, R. G., \& LAMB, M. R. (1981). A classification and analysis of short-term retention codes in the pigeon. The Psychol. ogy of Learning and Motivation, 15, 51-79.

ROBERTS, W. A. (1979). Spatial memory in the rat on a hierarchical maze. Learning and Motivation, 10, 117-140.

ROBERTS, W. A. (1981). Retroactive inhibition in rat spatial memory. Animal Learning \& Behavior, 9, 566-574.

ROBERTS, W. A. (1984). Some issues in animal spatial memory. In $\mathrm{H}$. L. Roitblat, T. G. Bever, \& H. S. Terrace (Eds.), Animal cognition. Hillsdale, NJ: Erlbaum.

RoItblat, H. L. (1980). Codes and coding processes in pigeon shortterm memory. Animal Learning \& Behavior, 8, 341-351.

Shavalia, D. A., Dodge, A. M., \& Beatty, W. W. (1981). Timedependent effects on ECS on spatial memory in rats. Behavioral ana Neural Biology, 31, 261-273.

Spear, N. E., Smith, G. J., Bryan, R. G., Gordon, W. C., Timmons, R., \& ChISZAR, D. A. (1980). Contextual influences on the interaction between conflicting memories in the rat. Animal Learn. ing \& Behavior, 8, 273-281.

ZENTALL, T. R. (1970). Effects of context change on forgetting in rats. Journal of Experimental Psychology, 86, 440-448.

(Manuscript received July 24, 1984; revision accepted for publication December 10, 1984.) 